[Agr. Biol. Chem., Vol. 34, No. 6, p. 977 979, 1970]

\title{
Several Synthetic Hydroxy-acids as Plant Growth Regulators
}

Sir:

In 1965 Tamura and Chang ${ }^{11}$ isolated L- $\beta$ phenyllactic acid as a root growth promoter for rice and lettuce seedlings from culture filtrates of Exobasidium symploci-japonicae which is a fungus pathogenic for Symplopos japonica. Subsequently, we noticed that a few hydroxyacids produced by a fungus of Alternaria species markedly accelerated root elongation. ${ }^{2 \prime}$ These observations led us to synthesize various kinds of hydroxy-acids to test their biological activities. As the result, several compounds were found to exhibit marked root growth promotion which is preliminarily reported in this paper.

All of the hydroxy-acids tested here were known compounds and, unless commercially available, they were prepared according to the following methods:

a) Treatment of amino acids with nitrous acid.

b) Reformatskii reaction of carbonyl compounds followed by alkali hydrolysis.

c) Addition of hydrogen cyanide to carbonyl compounds followed by acid hydrolysis.

d) Treatment of long-chain unsaturated fatty acids with potassium permanganate or perbenzoic acid followed by acid hydrolysis resulting in the formation of cis- or transdihydroxy-acids.

The crude products thus obtained were purified by distillation under reduced pressure, silicic acid adsorption chromatography or recrystallization.

Biological activities were examined at first

1) S. Tamura and C. F. Chang, Agr. Biol. Chem., 29, 255 (1965).

2) The structures of these acids are under investigation. on the root growth of lettuce seedlings by the method of Frankland and Wareing. ${ }^{3}$ Subsequently, the compounds which exhibited marked promotion were subjected to the "Azukia rooting test" as well as the Avena coleoptile straight-growth test. In the Azukia test devised by Mitsuhashi et al.," we used epicotyl cuttings without disbudding which were dipped in test solutions for the first $36 \mathrm{hr}$. Numbers of adventitious roots protruding from cuttings were counted seven days after preparation. A typical feature of rooting is shown in Fig. 1.

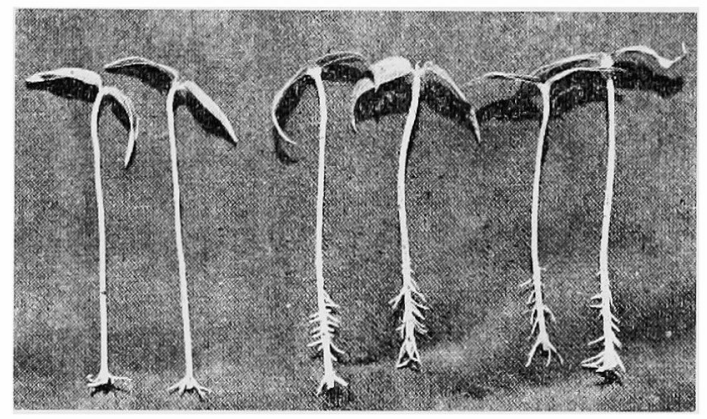

FIG. 1. Promotion on Root Formation of Azukia Cuttings by $\alpha$-Hydroxy- $\beta$-methylvaleric Acid (22) and $\beta$-Hydroxy-isocaproic Acid (25).

Left, control; middle, 25, $300 \mathrm{mg} /$ liter; right, 22, $300 \mathrm{mg} /$ liter.

Effects of aromatic hydroxy-acids including $\beta$-phenyllactic acid (4) on lettuce seedling roots are illustrated in Table I. The activities of 4-hydroxy-3-methoxymandelic acid (3) and $\alpha$-hydroxy- $\gamma$-phenylbutyric acid (6) were almost equal to that of 4 . Introduction of

3) B. Frankland and P. E. Wareing, Nature, $\mathbf{1 8 5}$, 255 (1960).

4) M. Mitsuhashi, H. Shibaoka and M. Shimokoriyama, Plant and cell Physiol., 10, 715 (1969). 
TABLE I. EFfeCtS OF AROMATIC HYDROXY-ACrDS ON THE RoOT GROWTH OF LETTUCE SEEDLINGS

Control $=100 ; 20.4 \mathrm{~mm}$

\begin{tabular}{|c|c|c|c|c|c|c|c|}
\hline & \multirow{2}{*}{ Compound ${ }^{a}$} & \multirow{2}{*}{ Method $^{b y}$} & \multicolumn{5}{|c|}{ Concentration (mg/liter) } \\
\hline & & & 10 & 30 & 100 & 300 & 600 \\
\hline 1 & Mandelic acid & & 113 & 118 & 91 & 27 & 0 \\
\hline 2 & $p$-Hydroxymandelic acid & & 108 & 106 & 137 & 127 & 96 \\
\hline $\mathbf{3}$ & 4-Hydroxy-3-methoxymandelic acid & & 109 & 125 & 164 & 170 & 144 \\
\hline 4 & $\beta$-Phenyllactic acid & a & 92 & 117 & 124 & 158 & 132 \\
\hline $\mathbf{5}$ & $\beta$-Hydroxy- $\beta$-phenylpropionic acid & $\mathrm{b}$ & 103 & 92 & 65 & 55 & 0 \\
\hline 6 & $\alpha$-Hydroxy-rmphenylbutyric acid & c & 102 & 136 & 188 & 101 & 13 \\
\hline
\end{tabular}

a) Purity of each compound was verified with gas chromatography or spectrometry. The behavior of the hydroxy-acids in the both analyses will be disclosed afterwards.

b) See the text.

TABLE II. EFFECTS OF AlIPHATIC HydRoXY-ACIDS ON THE ROOT GROWTH of LetTuce Seedlings

Control $=100 ; 20.5 \mathrm{~mm}$

\begin{tabular}{|c|c|c|c|c|c|c|c|}
\hline & \multirow{2}{*}{ Compound ${ }^{a}$ ) } & \multirow{2}{*}{ Method $^{b 1}$} & \multicolumn{5}{|c|}{ Concentration (mg/liter) } \\
\hline & & & 10 & 30 & 100 & 300 & 600 \\
\hline 7 & Glycolic acid & & 98 & 78 & 69 & 18 & 0 \\
\hline 8 & $\beta$-Hydroxypropionic acid & & 110 & 101 & 97 & 61 & 18 \\
\hline 9 & Tartronic acid & & 69 & 69 & 68 & 57 & 44 \\
\hline 10 & $\alpha$-Hydroxy-n-butyric acid & a & 102 & 95 & 91 & 78 & 30 \\
\hline 11 & $\alpha$-Hydroxy-isobutyric acid & & 103 & 111 & 113 & 100 & 20 \\
\hline 12 & $\beta$-Hydroxy-n-butyric acid & $\mathrm{b}$ & 103 & 104 & 125 & 104 & 20 \\
\hline 13 & $\alpha$-Hydroxy-n-valeric acid & a & 125 & 148 & 177 & 111 & 105 \\
\hline 14 & $\alpha$-Hydroxy- $\beta$-methylbutyric acid & $\mathrm{a}$ & 100 & 100 & 106 & 105 & 63 \\
\hline 15 & $\beta$-Hydroxy-n-valeric acid & $\mathrm{b}$ & 97 & 107 & 124 & 159 & 81 \\
\hline 16 & $\beta$-Hydroxy-isovaleric acid & $\mathrm{b}$ & 94 & 98 & 86 & 32 & 0 \\
\hline 17 & $\alpha$-Hydroxy- $\alpha$-methylbutyric acid & c & 104 & 100 & 84 & 38 & 0 \\
\hline 18 & L- $\alpha, \delta$-Dihydroxyvaleric acid & a & 106 & 110 & 141 & 147 & 17 \\
\hline 19 & $\alpha$-Hydroxy-n-caproic acid & $\mathbf{a}$ & 157 & 169 & 121 & 83 & 0 \\
\hline 20 & $\alpha$-Hydroxy-isocaproic acid & a & 93 & 118 & 109 & 90 & 11 \\
\hline 21 & $\alpha$-Hydroxy- $\alpha$-methylvaleric acid & $c$ & 98 & 98 & 92 & 93 & 67 \\
\hline 22 & $\alpha$-Hydroxy- $\beta$-methylvaleric acid & a & 116 & 141 & 138 & 87 & 3 \\
\hline 23 & $\alpha$-Hydroxy- $\alpha$-methyl-isovaleric acid & $\mathrm{c}$ & 101 & 108 & 117 & 94 & 45 \\
\hline 24 & $\beta$-Hydroxy-n-caproic acid & $\mathrm{b}$ & 116 & 103 & 135 & 139 & 27 \\
\hline 25 & $\beta$-Hydroxy-isocaproic acid & $\mathrm{b}$ & 112 & 108 & 147 & 100 & 14 \\
\hline 26 & $\beta$-Hydroxy- $\alpha$-methylvaleric acid & $\mathrm{b}$ & 106 & 127 & 204 & 168 & 34 \\
\hline 27 & $\beta$-Hydroxy- $\beta$-methylvaleric acid & $\mathrm{b}$ & 105 & 108 & 119 & 35 & 5 \\
\hline 28 & L-2,6-Dihydroxycaproic acid & a & 118 & 118 & 148 & 156 & 71 \\
\hline 29 & 2-Isopropylmalic acid & $c$ & 136 & 146 & 195 & 121 & 81 \\
\hline 30 & $\alpha$-Hydroxycaprylic acid & $\mathrm{a}$ & 104 & 114 & 126 & 135 & 127 \\
\hline 31 & $\beta$-Hydroxymyristic acid & & 92 & 85 & 85 & 74 & 67 \\
\hline 32 & 12-Hydroxystearic acid & & 99 & 94 & 86 & 78 & 72 \\
\hline 33 & Ricinoleic acid & & 104 & 100 & 101 & 105 & 96 \\
\hline 34 & cis-9,10-Dihydroxystearic acid & $\mathrm{d}$ & 93 & 96 & 101 & 102 & 116 \\
\hline 35 & trans $-9,10-D i h y d r o x y s t e a r i c$ acid & $\mathrm{d}$ & 101 & 98 & 98 & 102 & 112 \\
\hline
\end{tabular}

a) and $b_{1}$ cf. Table 1 . 
hydroxy or/and methoxy groups into the benzene ring of mandelic acid (1) distinctly increased the efficacy.

As shown in Table II, several aliphatic hydroxy-acids $(13,15,18,19,25,26,28$ and 29$)$ caused remarkable root elongation of lettuce seedlings indicating that the presence of benzene ring may not be required for the activity. On the other hand, some hydroxyacids $(\mathbf{7}, 9,16,17,31$ and 32$)$ listed in Table II exhibited suppression.

The activities of aliphatic and aromatic hydroxy-acids to promote the rooting of Azukia cuttings are shown in Table III. Several

TABLE III. EFFECTS OF HYDROXY ACIDS ON THE Rooting of Azukia Cuttings

Control $=100 ;$ Average 4.4 adventitious roots/cutting

\begin{tabular}{|c|c|c|c|c|c|}
\hline \multirow{2}{*}{$\begin{array}{l}\text { Com- } \\
\text { pound }\end{array}$} & \multicolumn{5}{|c|}{ Concentration (mg/liter) } \\
\hline & 10 & 30 & 100 & 300 & 600 \\
\hline 1 & 104 & 98 & 231 & 238 & 186 \\
\hline 2 & 88 & 94 & 98 & 137 & 133 \\
\hline 3 & 98 & 116 & 111 & 151 & 90 \\
\hline 4 & 93 & 116 & 127 & 153 & 24 \\
\hline 5 & 102 & 99 & 114 & 112 & 140 \\
\hline 6 & 113 & 120 & 211 & 242 & 170 \\
\hline 12 & 79 & 98 & 95 & 111 & 133 \\
\hline 14 & 125 & 145 & 149 & 131 & 130 \\
\hline 15 & 120 & 128 & 126 & 169 & 135 \\
\hline 18 & 77 & 73 & 113 & 154 & 220 \\
\hline 19 & 84 & 193 & 193 & 120 & 102 \\
\hline 20 & 93 & 98 & 100 & 123 & 113 \\
\hline 22 & 82 & 86 & 144 & 276 & 348 \\
\hline 24 & 135 & 140 & 173 & 271 & 114 \\
\hline 25 & 133 & 207 & 233 & 405 & 451 \\
\hline 26 & 96 & 140 & 319 & 120 & 120 \\
\hline 27 & 96 & 100 & 163 & 333 & 412 \\
\hline 28 & 90 & 93 & 105 & 166 & 163 \\
\hline 29 & 105 & 126 & 190 & 203 & 157 \\
\hline 33 & 71 & 95 & 101 & 131 & 119 \\
\hline
\end{tabular}

hydroxy-acids $(\mathbf{1}, 6,22,24,25,26,27$ and 29$)$ showed remarkable promotion of adventitious root formation. Among these, only 6, 25, 26 and 29 were effective in root elongation of lettuce seedlings as described above.

Contrary to the expectation, no hydroxy-
TABLE IV. FFFECTS OF HYDROXY-ACIDS ON THE ELONGATION OF Avena Coleoptile Segments

Control $=100 ; 6.24 \mathrm{~mm}$

\begin{tabular}{lrrrrr}
\hline Com- & \multicolumn{6}{c}{ Concentration } & (mg/liter) & \\
pound & 10 & 30 & 100 & 300 & 600 \\
\hline $\mathbf{4}$ & 98 & 101 & 100 & 98 & 86 \\
$\mathbf{6}$ & 102 & 104 & 103 & 99 & 97 \\
$\mathbf{1 4}$ & 108 & 111 & 112 & 111 & 113 \\
$\mathbf{1 5}$ & 113 & 110 & 101 & 110 & 105 \\
$\mathbf{1 8}$ & 104 & 104 & 102 & 107 & 103 \\
$\mathbf{1 9}$ & 102 & 100 & 102 & 96 & 80 \\
$\mathbf{2 2}$ & 107 & 133 & 113 & 104 & 81 \\
$\mathbf{2 4}$ & 111 & 114 & 107 & 110 & 103 \\
$\mathbf{2 5}$ & 104 & 110 & 113 & 110 & 105 \\
$\mathbf{2 6}$ & 113 & 110 & 104 & 110 & 103 \\
$\mathbf{2 9}$ & 109 & 107 & 110 & 111 & 108 \\
$\mathbf{3 3}$ & 105 & 118 & 110 & 112 & 97 \\
IAA & $146(0.1 \mathrm{mg} /$ liter $)$ & & & \\
\hline
\end{tabular}

acids revealed activity in the Avena straightgrowth test as shown in Table IV. This suggests that the action mechanism of the hydroxy-acids on the roots may differ from that of auxins.

From the results so far obtained, it is impossible to propose definite conclusion on structure-activity relationships, but this paper would be the first one to report the comparative activities of synthetic hydroxy-carboxylic acids as root growth promoters.

We are grateful to Mr. H. Kaneko of Central Research Institute of Japan Monopoly Corporation for supplying several hydroxymacids.

\section{Yoichi Mrkami \\ Hiroyasu TAKAHARA \\ Hiroko IIMURA \\ Akinori SUZUKI* \\ Saburo TAMURA*}

Central Research Institute

Japan Monopoly Corporation

Shinagawa-ku, Tokyo

* Department of Agricultural Chemistry

The University of Tokyo

Bunkyo-ku, Tokyo

Received March 28, 1970 